\title{
Identification of intermediate-risk patients with acute symptomatic pulmonary embolism
}

\author{
Carlo Bova ${ }^{1}$, Olivier Sanchez ${ }^{2,3}$, Paolo Prandoni ${ }^{4}$, Mareike Lankeit $^{5}$, \\ Stavros Konstantinides ${ }^{5}$, Simone Vanni ${ }^{6}$ and David Jiménez ${ }^{7}$
}

Affiliations: 'Dept of Medicine, University Hospital of Cosenza, Cosenza, Italy. ${ }^{2}$ Université Paris Descartes, Sorbonne Paris Cité, Paris, France. ${ }^{3}$ Division of Respiratory and Intensive Care Medicine, Hôpital Européen Georges Pompidou, AP-HP, Paris, France. ${ }^{4}$ Dept of Medicine, Thromboembolism Unit, University Hospital of Padua, Padua, Italy. ${ }^{5}$ Center for Thrombosis and Hemostasis, University of Mainz, Mainz, Germany. ${ }^{6}$ Emergency Dept, Azienda Ospedaliero-Universitaria Careggi, Florence, Italy. ${ }^{7}$ Respiratory Dept, Ramón y Cajal Hospital, IRYCIS, Madrid, Spain.

Correspondence: David Jiménez, Respiratory Dept, Ramón y Cajal Hospital, IRYCIS, 28034 Madrid, Spain. E-mail: djc_69_98dyahoo.com

ABSTRACT The identification of normotensive patients with acute pulmonary embolism (PE) at high risk of adverse PE-related clinical events (i.e. intermediate-risk group) is a major challenge.

We combined individual patient data from six studies involving 2874 normotensive patients with PE. We developed a prognostic model for intermediate-risk PE based on the clinical presentation and the assessment of right ventricular dysfunction and myocardial injury. We used a composite of PE-related death, haemodynamic collapse or recurrent PE within 30 days of follow-up as the main outcome measure.

The primary outcome occurred in 198 (6.9\%) patients. Predictors of complications included systolic blood pressure 90-100 mmHg (adjusted odds ratio (aOR) 2.45, 95\% CI 1.50-3.99), heart rate $\geqslant 110$ beats per min (aOR 1.87, 95\% CI 1.31-2.69), elevated cardiac troponin (aOR 2.49, 95\% CI 1.71-3.69) and right ventricular dysfunction (aOR 2.28, 95\% CI 1.58-3.29). We used these variables to construct a multidimensional seven-point risk index; the odds ratio for complications per one-point increase in the score was 1.55 (95\% CI 1.43-1.68; $\mathrm{p}<0.001)$. The model identified three stages (I, II and III) with 30-day PE-related complication rates of $4.2 \%, 10.8 \%$ and $29.2 \%$, respectively.

In conclusion, a simple grading system may assist clinicians in identifying intermediate-risk PE.

@ERSpublications

A simple grading system to identify intermediate-risk pulmonary embolism http://ow.ly/uItnL

For editorial comments see page 565 .

Earn CME accreditation by answering questions about this article. You will find these at erj.ersjournals.com/misc/ cmeinfo.xhtml

Received: Jan 082014 | Accepted after revision: March 022014 | First published online: April 22014

Support statement: The study was supported by a grant from the Instituto de Salud Carlos III (PI11/00246). The funding source had no role in the study design, analysis or interpretation or in the decision to submit the manuscript for publication.

Conflict of interest: None declared.

Copyright @ERS 2014 


\section{Introduction}

As patients with acute symptomatic pulmonary embolism (PE) who present with arterial hypotension or shock have a high risk of death, treatment guidelines suggest consideration of thrombolysis for such patients [1-3]. For haemodynamically stable patients with PE, the categorisation of risk for subgroups may assist with decision making regarding therapy [4].

While most haemodynamically stable patients with PE have a good prognosis when adequately treated with standard anticoagulation, some evidence suggests that a subset of normotensive patients with acute symptomatic PE (i.e. intermediate-risk group) may have a reasonable risk/benefit ratio for thrombolytic therapy. However, single markers of right ventricular (RV) dysfunction (e.g. echocardiography, spiral computed tomography (CT) or brain natriuretic peptide testing (BNP)) and myocardial injury (e.g. cardiac troponin $\mathrm{T}$ or I testing) have an insufficient positive predictive value for PE-specific complications to drive decision making toward such therapy [5]. In the absence of explicit clinical prediction rules that identify this subgroup of patients, the American College of Chest Physicians guidelines suggested that such patients should be identified predominantly by clinical evidence of instability (e.g. a decrease in systolic blood pressure (SBP) to a value $>90 \mathrm{mmHg}$, tachycardia, elevated jugular venous pressure, clinical evidence of poor tissue perfusion and hypoxaemia) [1]. Thus, fine tuning risk stratification for normotensive patients with acute symptomatic PE necessitates further development of multimarker prognostic models [6].

By increasing the number of patients studied and by enabling adjustment for confounding variables, metaanalysis of individual patients' data from several studies has the potential to answer questions that individual trials and meta-analyses of aggregate data from such cohorts cannot answer [7]. For this reason, authors of six studies pooled individual patients' data from their studies that evaluated the prognostic usefulness of imaging and laboratory data in normotensive patients with acute symptomatic PE [5, 8-12]. This study aimed to develop a method to predict PE-related complications based on the clinical presentation and the assessment of RV dysfunction and injury.

\section{Methods}

A core group of investigators (C. Bova and D. Jiménez) developed the process for obtaining patient level data and conducting analyses, and all the co-authors approved them before the data collection phase.

\section{Source study characteristics}

The six included studies had the following characteristics: original publication; prospective cohort study of patients with an objectively confirmed diagnosis of acute symptomatic PE; assessment of RV dysfunction either by echocardiogram or CT; measurement of markers of myocardial injury; and analysis of PE-related complications within the cohort of normotensive patients.

Source study quality assessment

We used a modified Newcastle-Ottawa scale to assess the quality of nonrandomised studies [13]. We evaluated the studies on the basis of the selection and outcome criteria alone, as the availability of patientlevel data rendered selection criteria irrelevant. High-quality studies had a score of $\geqslant 4$ points.

\section{Development of individual patient database}

We contacted the principal investigator of each eligible study to explain the meta-analysis objectives and analysis plan. After all investigators agreed to share their databases and appropriate permissions were obtained, the databases were transferred to a central location under the auspices of two reviewers. Data were checked, explanations for coding and uncertain or missing data were clarified, and a single pooled database was developed.

\section{Patient population}

The study population comprised normotensive patients (defined as a SBP $>90 \mathrm{mmHg}$ ) with an objectively confirmed diagnosis of acute symptomatic PE who initially received standard anticoagulation. The study excluded patients who had treatment with thrombolytics at the time of PE diagnosis. For two included studies that enrolled haemodynamically unstable patients $[9,10]$, those patients were excluded from our analyses. All studies [5, 8-12] assessed RV dysfunction by echocardiography and investigators used these data to develop the prediction rule. Echocardiographic criteria of RV dysfunction were similar across studies (table 1). The PROTECT (Prognostic Value of CT Scan in Haemodynamically Stable Patients with Acute Symptomatic PE) study also assessed RV dysfunction by CT and used a predefined cut-off point to determine abnormal (RV dysfunction) versus normal (non-RV dysfunction) [12]. Although not available in the original reports, data on CT-assessed RV dysfunction were also available in the databases of two studies $[9,10]$. For those patients 


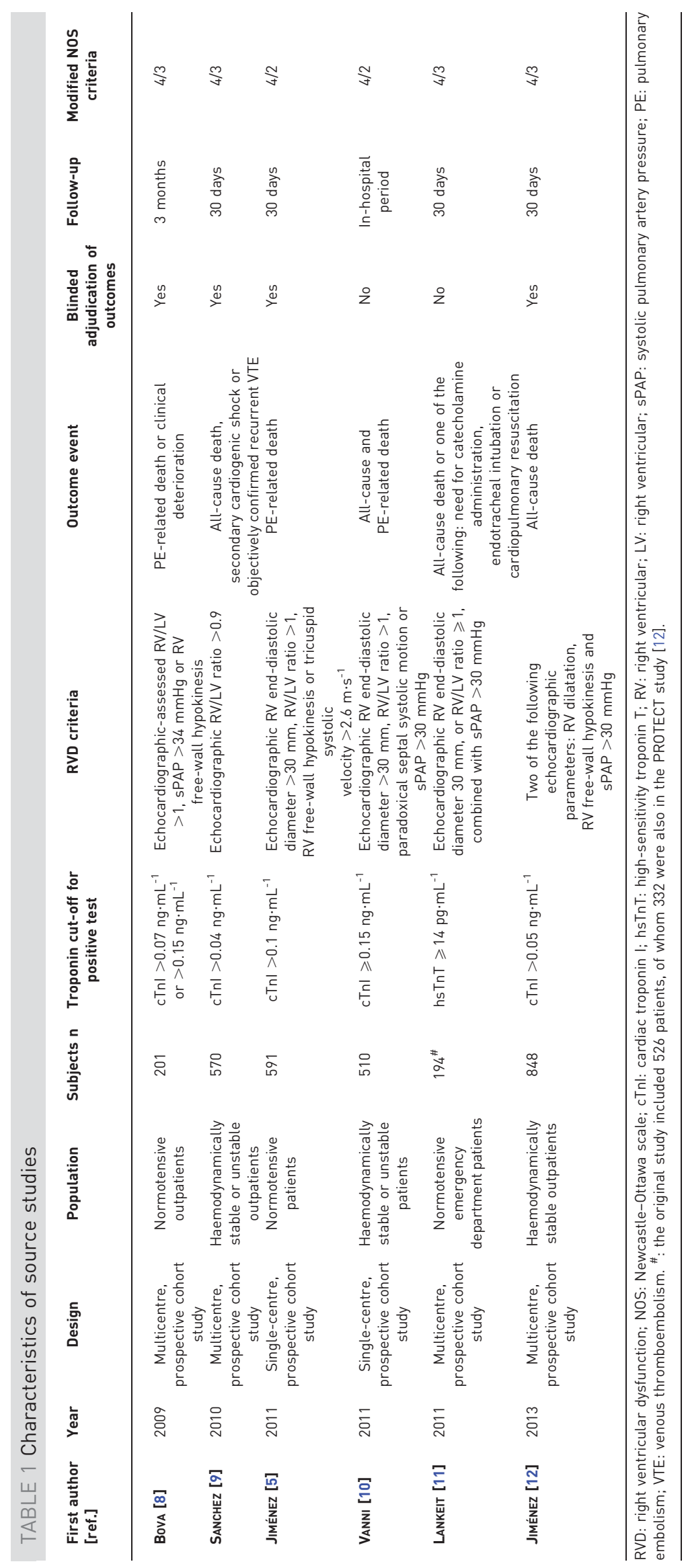


enrolled in the study without echocardiographic data from around the time of PE diagnosis, data on CT-assessed RV dysfunction were considered for the pooled analysis.

Different biomarkers for myocardial injury and different cut-off points for abnormal levels were used among the studies. For the troponin I studies [5, 8-10, 12], investigators used different manufacturers' assays and different cut-off points. In most of the studies, the cut points for troponin assays were defined according to standard criteria, which were values exceeding the $99 \%$ percentile of healthy subjects with a coefficient of variation of $10 \%$. One study assessed the prognostic value of a high-sensitivity troponin assay [11].

\section{Patient follow-up and outcomes}

This study used a composite outcome of PE-related complications that consisted of death from PE, haemodynamic collapse or adjudicated recurrent nonfatal PE through 30 days after the diagnosis of PE as the primary endpoint. Though one study reported in-hospital outcomes [10], investigators were able to provide complete 30-day follow-up for their patients. PE was considered the cause of death if there was objective documentation or if the cause was unexplained and PE could not be confidently ruled out. The study protocol defined haemodynamic collapse as SBP $<90 \mathrm{mmHg}$ for $\geqslant 15 \mathrm{~min}$, need for catecholamine administration because of persistent arterial hypotension or shock, need for thrombolysis, need for endotracheal intubation or need for cardiopulmonary resuscitation. Recurrent PE was confirmed either by the presence of a new intraluminal filling defect, by an extension of a previous filling defect on CT or by a new perfusion scan defect involving $\geqslant 75 \%$ of a lung segment. In addition, the study selected in-hospital PE-related complications and 30-day PE-related mortality as the secondary end-points.

\section{Statistical analysis}

In order to quantify unexplained study heterogeneity, we calculated the median odds ratio (MOR). To account for the effects of clustering of patients within studies, we used a mixed-effect regression model. Candidate variables that were associated with 30-day PE-related complications on univariate analysis $(\mathrm{p}<0.20)$ were included as potential covariates in a multiple logistic regression model [14]. Variable selection in multivariable modelling was based on clinical and statistical significance [15]. We assessed performance of the model by the area under the receiver operating characteristic (ROC) curve [16], and evaluated model calibration with the modified Hosmer-Lemeshow Chi-squared statistic, where values $<20$ indicate good calibration [14]. The model for 30-day complications was assessed for possible over-fit using linear shrinkage estimators [17, 18].

A score-based prediction rule for the primary end-point at 30 days was developed from the logistic regression model by using a regression coefficient-based scoring method [19, 20]. Integer scores were assigned by dividing risk-factor coefficients by the lowest coefficient and rounding up to the nearest unit for categorical variables [21]. The overall risk score was calculated by adding each component together. We validated the 30-day end-point model internally using the bootstrap in the derivation dataset by sampling with replacement for 200 iterations [22-24].

Statistical significance was defined as a two-tailed p-value of $<0.05$ for all analyses. Analyses were performed using SPSS version 15.0 for Windows (SPSS, Inc., Chicago, IL, USA) while we used Stata version 12.1 for Windows (StataCorp, College Station, TX, USA) for developing the mixed-effect regression model.

\section{Results}

\section{Source study and patient characteristics}

Table 1 shows the derivation of the source studies used in this meta-analysis. There were 2874 normotensive patients in the cohort and approximately two thirds were females. Table 2 describes presenting features (vital signs and initial laboratory values) and frequency of comorbid conditions of the cohort.

Of the 2693 patients with troponin measurements in the cohort, 988 (37\%, 95\% CI 35-39\%) had myocardial injury and 794 (50\%, 95\% CI 47-52\%) out of 1590 had an elevated BNP level. Investigators performed a transthoracic echocardiogram in 2188 (76\%) patients. Of these, 716 (33\%, 95\% CI 31-35\%) were diagnosed with RV dysfunction. On CT evaluation, 787 (50\%, 95\% CI 48-53\%) out of 1571 patients had RV dysfunction (defined as RV/left ventricular ratio >1). Overall, 910 (36\%, 95\% CI 34-38\%) out of 2504 patients had RV dysfunction assessed either by echocardiogram or CT.

\section{Source study quality assessment and study heterogeneity}

All included studies were of high quality according to the Newcastle-Ottawa scale (table 1) and an analysis evaluating the effect of differences in study quality was not needed. The MOR was 1.62 and suggested that the study heterogeneity was large. 
TABLE 2 Study patient characteristics

Derivation cohort

\begin{tabular}{|c|c|}
\hline \multirow{2}{*}{\multicolumn{2}{|c|}{$\begin{array}{l}\text { Subjects } \mathbf{n} \\
\text { Clinical characteristics }\end{array}$}} \\
\hline & \\
\hline Age years median (interquartile range) & $72(60-80)$ \\
\hline Age $>80$ years & 675 (23) \\
\hline Males & 1122 (39) \\
\hline \multicolumn{2}{|l|}{ Risk factors for VTE } \\
\hline Cancer ${ }^{\#}$ & 551 (19) \\
\hline Recent surgery & $311(11)$ \\
\hline Previous VTE & $424(15)$ \\
\hline Immobilisation ${ }^{+}$ & $475(16)$ \\
\hline \multicolumn{2}{|l|}{ Comorbid diseases } \\
\hline COPD & $381(13)$ \\
\hline Chronic heart failure & $212(7.4)$ \\
\hline \multicolumn{2}{|l|}{ Clinical symptoms and signs at presentation } \\
\hline Syncope & $349(12)$ \\
\hline Chest pain & $1170(41)$ \\
\hline Dyspnoea & $2062(72)$ \\
\hline Heart rate $\geqslant 110$ beats per $\min ^{\S}$ & $533(18)$ \\
\hline $\mathrm{SaO}_{2}<90 \%^{f}$ & $625(22)$ \\
\hline SBP $90-100 \mathrm{mmHg}$ & $156(5.4)$ \\
\hline \multicolumn{2}{|l|}{ Simplified PESI } \\
\hline Low-risk & $1029(36)$ \\
\hline High-risk & $1845(64)$ \\
\hline \multicolumn{2}{|l|}{ Imaging and cardiac biomarkers } \\
\hline Echocardiographic RV dysfunction ${ }^{\top \uparrow,++}$ & 716 (33) \\
\hline CT-assessed RV dysfunction ${ }^{\S \S, f f}$ & $787(50)$ \\
\hline RV dysfunction $\bullet^{\top}, \# \# \#, \uparrow \uparrow$ & $910(36)$ \\
\hline $\mathrm{BNP}>100 \mathrm{pg} \cdot \mathrm{mL}^{-1+++}$ & $794(50)$ \\
\hline Elevated cardiac troponin ${ }^{\bullet \uparrow, \S \S \S}$ & 988 (37) \\
\hline \multicolumn{2}{|l|}{ Treatment } \\
\hline Insertion of an IVC filter & $63(2.2)$ \\
\hline
\end{tabular}

Data are presented as $\mathrm{n}(\%)$ unless otherwise stated. VTE: venous thromboembolism; COPD: chronic obstructive pulmonary disease; $\mathrm{SaO}_{2}$ : arterial oxyhaemoglobin saturation; SBP: systolic blood pressure; PESI: Pulmonary Embolism Severity Index; RV: right ventricular; CT: computed tomography; BNP: brain natriuretic peptide; IVC: inferior vena cava. ${ }^{\#}$ : active or under treatment in the previous year; ${ }^{\circ}$ : in the previous month; ${ }^{+}$: nonsurgical patients who had been immobilised (i.e. total bed rest with bathroom privileges) for $\geqslant 4$ days in the month prior to pulmonary embolism diagnosis; ${ }^{\S}: n=2743 ;{ }^{f}: n=2429 ;{ }^{\# \#}: n=2742 ;{ }^{*}{ }^{\text {}}$ : see definitions, tests and cut-offs for specific studies in table $1 ;{ }^{++}: n=2188 ;{ }^{\S \S}$ : defined as right ventricle/left ventricle ratio $>1$;

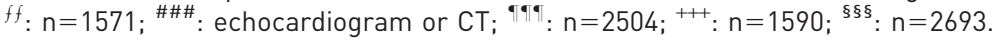

\section{Outcomes}

The study had complete primary outcome information for all patients at the end of the 30-day follow-up. The primary end-point occurred in $198(6.9 \%, 95 \%$ CI $6.0-7.8 \%)$ of the 2874 normotensive patients with acute symptomatic PE who entered the study. PE-related complications were due to PE-related death in 90 patients, haemodynamic collapse in 122 patients and nonfatal symptomatic recurrent PE in 18 patients (table 3). Of the $176(6.1 \%, 95 \%$ CI 5.2-7.0\%) out of 2874 patients who died during the 30-day follow-up period, each of the studies' adjudication committees considered death as "definitely" or "possibly" due to fatal PE in 90 out of 176 of these patients (51\% of the total deaths, 95\% CI 44-59\%), and this corresponded to a cumulative rate of definite or possible fatal PE of $3.1 \%$ (95\% CI, 2.5-3.8\%) at 30 days after PE diagnosis. $178(6.1 \%, 95 \%$ CI $5.3-7.1 \%)$ out of 874 patients experienced the primary end-point during hospitalisation. In-hospital PE-related complications were due to PE-related death in $78(2.7 \%$, $95 \%$ CI $2.1-3.3 \%)$ out of 874 patients.

\section{Predictors of 30-day PE-related complications}

The study assessed predictors for 30-day PE-related complications in 2216 patients who had complete baseline data required for this study. Results of univariate analysis for all potential predictors are shown in table 4. Multivariable model for 30-day PE-related complications is shown in table 4. There was no statistical evidence of over-fit as demonstrated by linear shrinkage estimation in the multivariable model $(r=0.985)$. 
TABLE 3 Pulmonary embolism (PE)-related complications in the cohort of normotensive patients with acute symptomatic PE during 30 days of follow-up

Patients

\begin{tabular}{lc} 
Cohort $\mathbf{n}$ & 2874 \\
PE-related complications & $\#$ \\
All-cause death & $198(6.9)$ \\
PE-related death & $176(6.1)$ \\
Haemodynamic collapse & $90(3.1)$ \\
Nonfatal recurrent PE & $122(4.2)$ \\
\hline
\end{tabular}

Data are presented as $\mathrm{n}(\%)$ unless otherwise stated. ${ }^{\#}$ : PE-related mortality, haemodynamic collapse or recurrent $\mathrm{PE}$; patients may have more than one event fulfilling the definition of PE-related complications. ": at least one of systolic blood pressure $<90 \mathrm{mmHg}$ for $\geqslant 15 \mathrm{~min}$, need for catecholamine administration because of persistent arterial hypotension or shock, need for endotracheal intubation, or need for cardiopulmonary resuscitation.

Model predictors of PE-related complications through 30-day after the diagnosis of PE included SBP (adjusted odds ratio (aOR) 2.45, 95\% CI 1.50-3.99), heart rate (aOR 1.87, 95\% CI 1.31-2.69), myocardial injury (aOR 2.49, 95\% CI 1.71-3.61) and RV dysfunction (aOR 2.28, 95\% CI 1.58-3.29). Although syncope was a significant predictor of complications, it was correlated with SBP (Pearson $r=0.15$ ) and was no longer significant when SBP was entered into the model (table 4). Notably, although age and immobilisation were associated with the primary end-point in univariate analysis (table 4), there was no significant effect after adjustment for other factors in the multivariable model $(\mathrm{p}>0.20)$.

\section{Risk score}

Patients with higher risk scores were at higher risk of PE-specific complications; the odds ratio for complications per one-point increase in the score was 1.55 (95\% CI 1.43-1.68, p < 0.001). Points were assigned to variable categories to create a point-score model (range 0-7) for prediction of PE-related complications, as shown in table 5. The total point scores were used to classify patients as stage I (0-2 points), stage II (3-4 points) or stage III ( $>4$ points). Cumulative incidence of 30-day PE-related complications differed substantially among stages for the model $(4.2 \%, 10.8 \%$, and $29.2 \%$ for stages I, II and III, respectively) (fig. 1 and table 6). Similarly, a graded increase in risk occurred when investigators assessed in-hospital PE-related complications and 30-day PE-related mortality. The in-hospital complication rate

TABLE 4 Factors associated with 30-day pulmonary embolism-related complications in 2216 normotensive patients with acute symptomatic pulmonary embolism

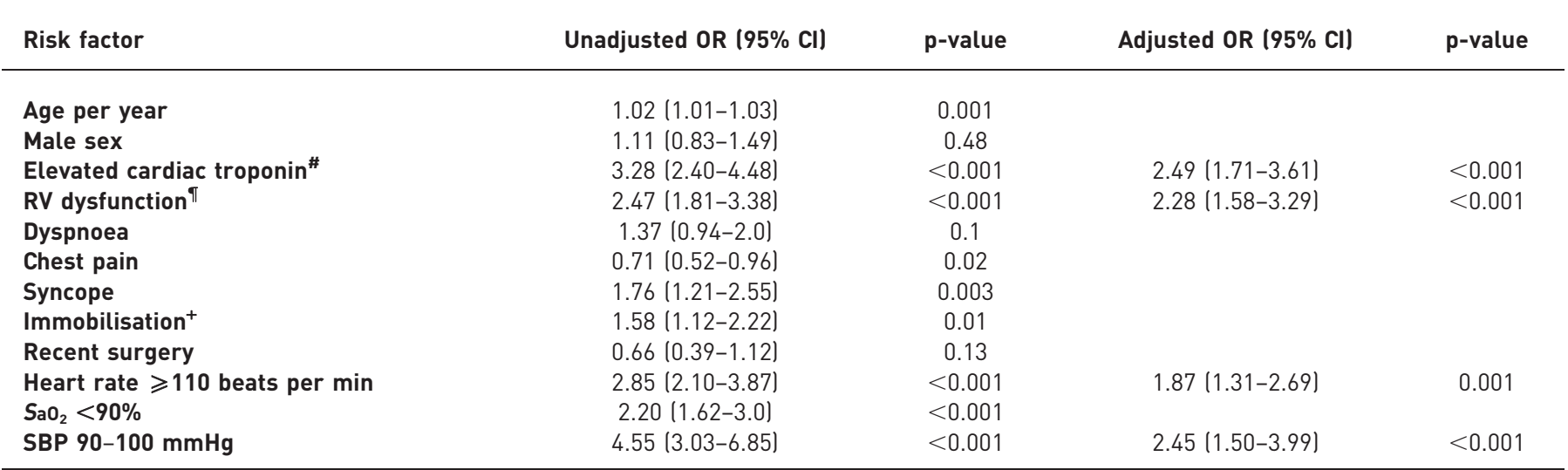

Hosmer-Lemeshow goodness-of-fit test statistic was used for the complete case analysis: Chi-squared (8 degrees of freedom) 2.34, $p=0.80$. RV: right ventricular; $\mathrm{SaO}_{2}$ : arterial oxyhaemoglobin saturation; SBP: systolic blood pressure. " : see definitions, tests and cut-offs for specific studies in table $1 ;$ " : assessed by echocardiogram or computed tomography (if echocardiogram not available); ${ }^{+}$: immobilised patients are defined in this analysis as nonsurgical patients who had been immobilised (i.e. total bed rest with bathroom privileges) for $\geqslant 4$ days in the month prior to pulmonary embolism diagnosis. 
TABLE 5 Risk score

Points are assigned for each variable of the scoring system to obtain a total point score (range 0-7). SBP: systolic blood pressure; RV: right ventricular; CT: computed tomography.

was 3.6\% for stage I, 9.7\% for stage II and $28.0 \%$ for stage III, while 30-day PE related mortality was $1.7 \%$, $5.0 \%$ and $15.5 \%$ for stages I, II and III, respectively (table 6).

\section{Model internal validation}

Analyses suggested that the final model had good predictive performance (table 7). In the derivation set $(n=2216)$, the area under the ROC curve was 0.73 (95\% CI 0.68-0.77) for 30-day PE-related complications. The final model was well calibrated (Hosmer-Lemeshow Chi-squared 2.34, $\mathrm{p}=0.80$ for the lack of fit). The bootstrap-corrected area under the ROC curve was 0.73 for the model. When the 30 -day model was applied to in-hospital complications in the derivation set, the area under the ROC curve was 0.74 .

\section{Discussion}

We devised a simple grading system for identifying intermediate-risk patients with confirmed acute symptomatic PE. We believe that this index is useful because it includes one domain that quantifies RV dysfunction, one that captures myocardial injury and two independent domains (SBP and heart rate) that express the clinical haemodynamic consequences of PE.

While clinical models (e.g. simplified Pulmonary Embolism Severity Index (sPESI)) [25] may accurately identify low-risk patients with confirmed PE who might be candidates for partial or complete outpatient treatment, intensive search continues for a group of normotensive patients deemed as having a high risk of adverse PE-related clinical events (i.e. intermediate-risk group) that might benefit from an escalation of therapy (e.g. transfer to the intensive care unit or thrombolysis). However, a validated explicit clinical prediction rule that identifies this subgroup of patients is lacking. Observational studies have suggested an incremental prognostic value of the association of markers of RV dysfunction and injury over either alone [26] or the combination of echocardiography with clinical scores [27]. The present study validated and extended this concept. Indeed, we found that the combination of myocardial injury, RV dysfunction, tachycardia and mild hypotension predicted a seven-fold increase in the risk of an adverse 30-day PErelated outcome. Because of the risk of bleeding from thrombolytic therapy, the risk for complications for stage II patients (10.8\%) may not warrant immediate aggressive treatment (less severe intermediate risk). Thus, it seems cautious to suggest that clinicians should consider using all prognostic tests included in the model (stage III) for identifying the more severe intermediate-risk patients with acute PE who might benefit from recanalisation procedures. Of note, the predictive model only classified $5.8 \%$ of patients as stage III,

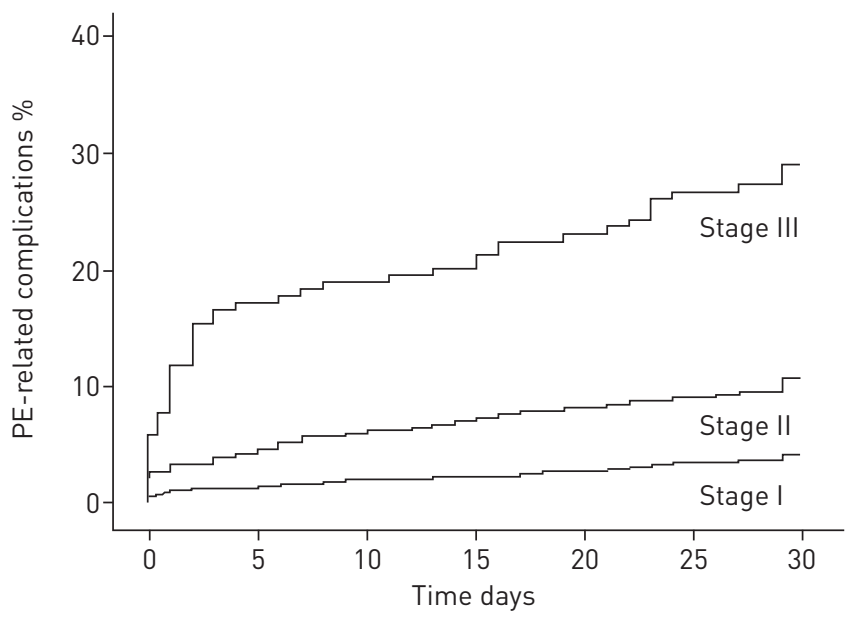

FIGURE 1 Cumulative pulmonary embolism (PE)-related complications stratified by stage for the risk index. 


\begin{tabular}{lccc} 
& & Stage & \\
\cline { 2 - 4 } & I & II & III \\
\hline Points & $0-2$ & $3-4$ & $>4$ \\
Patients \% & 75.5 & 18.6 & 5.8 \\
30-day PE-related complications \% & 4.2 & 10.8 & 29.2 \\
In-hospital PE-related complications \% & 3.6 & 9.7 & 28.0 \\
30-day PE-related mortality \% & 1.7 & 5.0 & 15.5 \\
\hline
\end{tabular}

The total point score from table 5 was used to classify patients as stage I (0-2 points), stage II (3-4 points) or stage III (>4 points). Model-predicted 30-day pulmonary embolism (PE)-related complications are shown by stage.

suggesting that a very small proportion of normotensive patients with acute symptomatic PE might benefit from specific advanced therapy.

The optimal management of intermediate-risk patients with acute PE is unclear. A previous clinical trial did not demonstrate any significant mortality reduction in hemodynamically stable patients with acute PE and RV dysfunction who received thrombolytic therapy [28]. The PEITHO (Pulmonary Embolism Thrombolysis) trial determined the efficacy and safety of early thrombolytic treatment in normotensive patients with RV dysfunction, as detected on an echocardiogram or CT scan, and evidence of myocardial injury, as indicated by a positive troponin test [29]. The results of the trial showed that thrombolytic therapy prevented haemodynamic decompensation but increased the risk of major haemorrhage and stroke. Since the trial's mortality rate was low, the study might have not included a "sick enough" population. Thus, our findings have practical implications. First, different studies support the applicability of the sPESI as a first step for stratifying normotensive patients with acute PE [25]. Our data suggest that clinicians should further stratify patients initially deemed high risk (due to tachycardia or hypotension) by the sPESI by imaging of the RV and cardiac biomarkers. Alternatively, normotensive patients with acute PE in association with RV dysfunction and myocardial injury should undergo further risk stratification to identify subgroups at the highest risk of clinical deterioration because they may benefit the most from the administration of thrombolytic therapy. Prospective studies should further address the safety and efficacy of early recanalisation procedures for intermediate-risk PE patients who are identified with the proposed criteria.

Strengths of our analysis that increase the precision and validity of its findings are that we included data from a large number of participants, baseline variables were recorded at enrolment rather than retrospectively, participants were followed prospectively and outcomes were accurately diagnosed by adjudication committees in most studies. Moreover, regardless the different methods and the cut-offs used to define myocardial injury and RV dysfunction, the prognostic performance of the model was similar across studies (data not shown).

\section{Study limitations}

Some methodological limitations may affect the findings and interpretation of this study. First, our findings are limited to the types of patients included in the studies that we used in this meta-analysis, and selection bias could have skewed the study sample. However, the broad range of patients with acute symptomatic PE

\section{TABLE 7 Model performance}

Variable

\begin{tabular}{lcc}
\cline { 2 - 3 } & Predicted & Observed \\
\hline C-index $(95 \% \mathrm{CI})$ & \multicolumn{2}{c}{0.73 (0.68-0.77) } \\
30-day complicated course \% & 4.1 & 4.2 \\
Stage I & 11.5 & 10.8 \\
Stage II & 28.2 & 29.2 \\
Stage III & & \\
\hline
\end{tabular}


from multiple medical centres, countries and treatment settings decrease the likelihood of the inclusion of a skewed population in this study. Moreover, we pre-specified the study selection criteria, to attempt to minimise such bias. Second, although the variables for the clinical decision rule were collected prospectively, this study was a retrospective analysis. In addition, the model did not address some other factors that affect decision making, such as bleeding risk. Finally, although assessment of thrombotic burden assists with risk stratification in patients with acute symptomatic PE [30], the study could not test the prognostic value of variables (e.g. concomitant deep vein thrombosis) that were not consistently available across studies.

\section{Conclusion}

Significant predictors of PE-related complications in normotensive patients presenting to the hospital with acute PE include cardiac dysfunction, myocardial injury, increased heart rate and reduced SBP. Besides its excellent predictive power with regard to outcome, the proposed predicted score is simple to calculate and requires no special equipment. This makes it a practical tool of potentially widespread applicability. The risk index provides estimates of risk that may assist clinicians in counselling patients and families and may guide clinical decision making.

\section{References}

1 Kearon C, Akl EA, Comerota AJ, et al. Antithrombotic therapy for VTE disease: antithrombotic therapy and prevention of thrombosis, 9th ed: American College of Chest Physicians evidence-based clinical practice guidelines. Chest 2012; 141: Suppl., e419S-e494S.

2 Jaff MR, Archer SL, Cushman M, et al. Management of massive and submassive pulmonary embolism, iliofemoral deep vein thrombosis, and chronic thromboembolic pulmonary hypertension: a scientific statement from the American Heart Association. Circulation 2011; 123: 1788-1830.

3 Torbicki A, Perrier A, Konstantinides SV, et al. Guidelines on the diagnosis and management of acute pulmonary embolism: the Task Force for the Diagnosis and Management of Acute Pulmonary Embolism of the European Society of Cardiology (ESC). Eur Heart J 2008; 29: 2276-2315.

4 Penaloza A, Roy PM, Kline J. Risk stratification and treatment strategy of pulmonary embolism. Curr Opin Crit Care 2012; 18: 318-325.

5 Jiménez D, Aujesky D, Moores L, et al. Combinations of prognostic tools for identification of high-risk normotensive patients with acute symptomatic pulmonary embolism. Thorax 2011; 66: 75-81.

6 Steering Committee. Single-bolus tenecteplase plus heparin compared with heparin alone for normotensive patients with acute pulmonary embolism, who have evidence of right ventricular dysfunction and myocardial injury: rationale and design of the Pulmonary Embolism Thrombolysis (PEITHO) trial. Am Heart J 2012; 163: 33-38.

7 Riley RD, Lambert PC, Abo-Zaid G. Meta-analysis of individual participant data: rationale, conduct, and reporting. BMJ 2010; 340: c221.

8 Bova C, Pesavento R, Marchiori A, et al. Risk stratification and outcomes in hemodynamically stable patients with acute pulmonary embolism: a prospective, multicentre, cohort study with three months of follow-up. J Thromb Haemost 2009; 7: 938-944.

9 Sanchez O, Trinquart L, Caille V, et al. Prognostic factors for pulmonary embolism: the PREP study, a prospective multicenter cohort study. Am J Respir Crit Care Med 2010; 181: 168-173.

10 Vanni S, Nazerian P, Pepe G, et al. Comparison of two prognostic models for acute pulmonary embolism: clinical vs. right ventricular dysfunction-guided approach. J Thromb Haemost 2011; 9: 1916-1923.

11 Lankeit M, Jiménez D, Kostrubiec M, et al. Predictive value of the high-sensitivity troponin $\mathrm{T}$ assay and the Simplified Pulmonary Embolism Severity Index in hemodynamically stable patients with acute pulmonary embolism: a prospective validation study. Circulation 2011; 124: 2716-2724.

12 Jiménez D, Lobo JL, Monreal M, et al. Prognostic significance of multidetector computed tomography in normotensive patients with pulmonary embolism: results of the PROTECT study. Thorax 2014; 69: 109-115.

13 Wells GA, Shea B, O'Connell D, et al. The Newcastle-Ottawa scale (NOS) for assessing the quality of nonrandomised studies in meta-analyses. www.ohri.ca/programs/clinical_epidemiology/oxford.htm Date last updated: 2011.

14 Hosmer DW, Lemeshow S. Applied Logistic Regression. 2nd Edn. New York, John Wiley \& Sons Inc., 2000.

15 Steyerberg EW, Eijkemans MJ, Harrell FE Jr, et al. Prognostic modelling with logistic regression analysis. Stat Med 2000; 19: 1059-1079.

16 Hanley JA, McNeil BJ. The meaning and use of the area under a receiver operating characteristic (ROC) curve. Radiology 1982; 143: 29-36.

Harrell FE Jr, Lee KL, Mark DB. Multivariable prognostic models. Stat Med 1996; 15: 361-387.

Van Houwelingen JC, Le Cessie S. Predictive value of statistical models. Stat Med 1990; 9: 1303-1325.

Tu JV, Naylor CD. Clinical prediction rules. J Clin Epidemiol 1997; 50: 743-744.

Moons KG, Harrell FE, Steyerberg EW. Should scoring rules be based on odds ratios or regression coefficients? J Clin Epidemiol 2002; 55: 1054-1055.

21 Fine MJ, Auble TE, Yealy DM, et al. A prediction rule to identify low-risk patients with community acquired pneumonia. N Engl J Med 1997; 336: 243-250.

22 Efron B, Tibshirani RJ. An Introduction to the Bootstrap. New York, Chapman \& Hall/CRC Press, 1998.

23 Steyerberg EW, Eijkemans MJ, Harrell FE Jr, et al. Prognostic modeling with logistic regression analysis: in search of a sensible strategy in small data sets. Med Decis Making 2001; 21: 45-56.

24 Steyerberg EW, Harrell FE Jr, Borsboom GJ, et al. Internal validation of predictive models. J Clin Epidemiol 2001; 54: 774-781.

25 Jiménez D, Moores L, Gómez V, et al. RIETE Investigators. Simplification of the pulmonary embolism severity index for prognostication in patients with acute symptomatic pulmonary embolism. Arch Intern Med 2010; 170: $1383-1389$. 

html\#!/3042/presentation/49220

30 Jiménez D, Aujesky D, Díaz G, et al. Prognostic significance of concomitant deep vein thrombosis in patients presenting with acute symptomatic pulmonary embolism. Am J Respir Crit Care Med 2010; 181: 983-991. 